Einführung zum Thema

Oper Orthop Traumatol 2020 · 32:385-386 https://doi.org/10.1007/s00064-020-00678-4

(c) Springer Medizin Verlag GmbH, ein Teil von Springer Nature 2020

Der Fixateur externe wird gerne als das „Arbeitspferd“ der Osteosynthese bezeichnet. Sowohl für diaphysäre Frakturen als auch für Gelenkfrakturen, für Weichteilverletzungen und -schäden, z. B. bei Verbrennungen, aber auch bei Infektionen kann der Fixateur externe eingesetzt werden.

Schaut man in PubMed zum Thema Fixateur externe, finden sich Tausende von Arbeiten. Die äußere Fixation ist ein altes Verfahren [1]. Der Franzose Malgaigne setzte äußere Fixierung an Olekranon und Kniescheibe ein, also an Gelenken. Lambotte befasste sich mit der äußeren Fixation [7]. Raoul Hoffmann hob bereits auf dem Schweizer Chirurgen Kongress 1938 die Vorteile des Fixateur externe, den er damals als „Rotules à os“ bezeichnete hervor: Möglichkeit der Reposition, Blutarmut und Ausnutzung der Ligamentotaxis [2]. Der russische Chirurg Gawriil Abramowitsch Ilizarov aus Kurgan/Sibirien hat mit seinem Ringfixateursystem eine ganze Schule gegründet [6]. Seit Jahrzehnten hat in Deutschland Dankward Höntzsch den oft vernachlässigten Fixateur externe in den Fokus der Forschung und Publikation gestellt [3-5]. Bei gelenküberschreitenden Fixateurmontagen sollte auf eine möglichst weit überbrückende Konstruktion geachtet werden, um Fragmente und Weichteile möglichst wenig zu stören [5].

Der Fixateur externe ist das Verfahren der Wahl im Rahmen der Damage-Control beim Polytrauma [8, 9, 14]. Auch als lokales Damage-Control-Verfahren kann dieses Verfahren bei schweren Weichteilverletzungen eingesetzt werden, bei Letzteren auch als definitives Verfahren. Bei Gelenkfrakturen wird der Fixateur externe als solitäres Verfahren

\title{
Klaus Dresing
}

Klinik für Unfallchirurgie, Orthopädie und Plastische Chirurgie, Universitätsmedizin Göttingen, Göttingen, Deutschland

\section{Fixateur externe - temporäre gelenkübergreifende Fixation}

in der Primärversorgung, als additives Verfahren zur internen Osteosynthese oder zur Ruhigstellung luxierter Gelenke nach Reposition eingesetzt. Bei der Fraktureinrichtung in Gelenknähe wirkt bei der Reposition oder Distinktion mit dem Fixateur extern die Ligamentotaxis als Hauptrepositionskraft auf die Fragmente.

Das scheinbar einfache Verfahren der Fixateur-extern-Osteosynthese setzt die eingehende Kenntnis der Anatomie voraus, um sog. sichere Zonen der Pin-Platzierung, insbesondere am distalen $\mathrm{Hu}$ merus, zu finden [10-13]. Nerven-, Gefäß- und Sehnenverletzungen lassen sich auf diese Weise weitgehend vermeiden, mindestens jedoch minimieren. Nach jeder Fixateur-externe-Montage ist im Verlauf auf die Pin-Pflege zu achten, um Infektionen und Lockerungen zu vermeiden.

Wir haben in diesem Themenheft den Einsatz des Fixateurs an den großen Gelenken, Ellenbogen, Hand und Kniegelenk meist als temporäre Fixation zum Kernthema gemacht. Die renommierten Autoren haben, unterstützt von den hervorragenden medizinischen Illustratoren, die 3 Bereiche beeindruckend dargestellt.

Der Fixateur externe am Ellenbogen findet als temporäre Stabilisierung des Ellenbogens als „Damage-Control-Verfahren“ Verwendung. Als Bewegungsfixateur dient er zur Protektion der rekonstruierten oder refixierten Bänder und der Fragmente. Bei persistenter Luxationsneigung des Gelenkes kann er auch als alleiniges Verfahren zum Einsatz kommen. Bei der Distraktionsarthrolyse bei eingesteiftem Ellenbogengelenk hilft der Fixateur ebenso sowie als Rettungsver- fahren bei multiplen Begleiterkrankungen im Rahmen der Frakturbehandlung.

Die Indikation zur temporären Fixation am Handgelenk mit einem gelenkübergreifenden Fixateur externe ist mannigfaltig vom „Damage Control“ beim Polytrauma mit oder ohne Weichteilschaden, Frakturen des körperfernen Unterarms, der Handwurzel, bei Weichteilschaden und Luxationen in der Handwurzel.

Der gelenkübergreifende Fixateur am Kniegelenk wird sowohl bei distalen Femur- als auch bei Tibiakopffrakturen mit oder ohne Weichteilschaden, temporärer Stabilisierung komplexer Frakturen des Kniegelenks, beim Polytrauma, bei Knieluxation eingesetzt.

Mit dem CME-Beitrag über den temporären Fixateur externe und das Weichteilmanagement am oberen Sprunggelenk wird ein Update über ein weiteres großes Gelenk sehr gut vorgestellt.

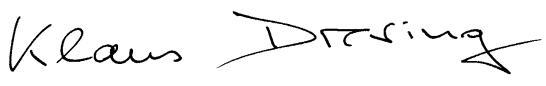

Prof. Dr. Klaus Dresing

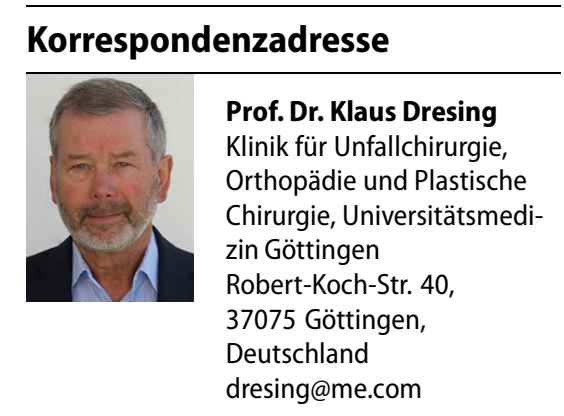

Interessenkonflikt. K. Dresing gibt an, dass kein Interessenkonflikt besteht. 


\section{Literatur}

1. Bisaccia M, Vicente $\mathrm{Cl}$, Meccariello Let al (2016) The history of external fixation, a revolution idea for the treatment of limb's traumatized and deformities: from Hippocrates to today. Can Open Orthop Traumatol J3:1-9

2. Hoffmann R(1938) "Rotulesà os" pourla"réduction dirigée", non sanglante, desfractures ("ostéotaxis"). Helv Med Acta 5:844

3. Höntzsch D (2010) Externalfixation osteosynthesis. Orthopade 39:192-200

4. Höntzsch D, Hunziker M (1994) A new open universal clamp for the external fixator tube system of the AO. Aktuelle Traumatol 24:24-29

5. Hontzsch D (2002) Joint-spanning and jointadjacent external fixators in the lower extremity (Gelenk übergreifende und gelenknahe Fixateurexterne-Montage an der unteren Extremität). Trauma Berufskrankh 4:394-398

6. Ilizarov GA (1990) Clinical application of the tension-stress effect for limb lengthening. Clin Orthop Relat Res 250:8-26

7. Lambotte A (1912) The operative treatment of fractures: report of fractures committee. Br Med J 2:1530

8. Maury C, Ramin S, Bonfils J, Dagneaux L, Faure $P$, Canovas F, Capdevila X, Charbit J (2020) Influence of a temporary stabilization device on respiratory status in patients with severe trauma with a femora shaft fracture treated by damage control strategy. Eur J Trauma Emerg Surg. https://doi.org/10.1007/ s00068-020-01300-9

9. Pfeifer R, Kalbas Y, Coimbra Retal (2020) Indications and interventions of damage control orthopedic surgeries: an expert opinion survey. Eur J Trauma Emerg Surg. https://doi.org/10.1007/s00068-020 01386-1

10. Plucknette BF, Tennent DJ, Hsu JR, Bates T, Burns TC (2020) Lateral external-fixation adjacent to radial nerve. Cureus 12:e7435

11. Sukegawa K, Kuniyoshi K, Suzuki T, Matsuura Y, Onuma K, Kenmoku T, Takaso M (2018) Effects of the elbow flexion angle on the radial nerve location around the humerus: a cadaver study for safe installation of a hinged external fixator.J Hand Surg Asian Pac 23:388-394

12. Suwanno $P$, Omokawa $S$, Nakanishi $Y$, Kira T, Tanaka Y (2020) Safe zone of pin insertion for nonbridging external fixators in distal radial fractures: MRI analysis. J Orthop Sci. https://doi. org/10.1016/j.jos.2019.12.005

13. Tang L, Yishake M, YeC, HadeH, LiZ,HeR(2020) Safe zone for lateral pin placement for external fixation of the distal humerus. Clin Anat 33:637-642

14. Tuttle MS, Smith WR, Williams AE, Agudelo JF, Hartshorn CJ, Moore EE, Morgan SJ (2009) Safety and efficacy of damage control external fixation versus early definitive stabilization for femoral shaft fractures in the multiple-injured patient. JTrauma 67:602-605

\section{Deutschlands größter Online-Event für Orthopäden und Unfallchirurgen \\ Digitale Woche O\&U \#digitaIOU2020}

Um den Sektionen, Arbeitsgruppen und Industriepartnern auch in diesem Jahr ihre Fortbildung und Produktpräsentationen zu ermöglichen, findet vom 19. bis 23. Oktober 2020 die Digitale Woche O\&U \#digitalOU2020 statt. Sie wird von der Akademie für Orthopädie und Unfallchirurgie (AOUC) organisiert. Die AOUC ist gemeinsames Referat des Berufsverbandes für Orthopädie und Unfallchirurgie (BVOU) und der Deutschen Gesellschaft für Orthopädie und Unfallchirurgie (DGOU).

Das täglich vierstündige kostenfreie OnlineFortbildungsprogramm setzt sich aus Podiumsdiskussionen, Online-Vorträgen, Videosessions, Live-Streams und vielen weiteren themenspezifischen Fortbildungsveranstaltungen zusammen. Jeder Tag hat einen wissenschaftlichen Schwerpunkt: Obere Extremität, Wirbelsäule, Becken, untere Extremität und Hüfte. Berufspolitische Diskussionen zu den Hot Topics IT und Datensicherheit, zu Video-Apps, zu Patientensteuerung durch die Kasse usw. ergänzen die wissenschaftlichen Formate.

„Die Ereignisse in diesem Jahr hat unser Fach zu Quantensprüngen in der Digitalisierung gezwungen. Nun gilt es die Vorteile zu nutzen und uns online auszutauschen", erklären die wissenschaftlichen Leiter Prof. Dr. Michael J. Raschke, Prof. Dr. Dieter C. Wirtz und Dr. Burkhard Lembeck.

Die Registrierung zu \#digitalOU2020 ist freigeschaltet. In den ersten 10 Tagen haben sich bereits 350 Teilnehmer angemeldet. Alle wichtigen Informationen zu Ablauf, Programm und Anmeldung sind unter der URL digitalOU.org abrufbar. An der Digitalen Woche O\&U \#digitalOU2020 kann per Computer oder über die O\&U Events App teilgenommen werden, die barrierefrei alle Formate wiedergibt.

\section{Über die AOUC}

Die Akademie für Orthopädie und Unfallchirurgie (AOUC) ist gemeinsames Referat des BVOU und der DGOU. Zweck ist der Austausch, die Koordination und die Bündelung von rein ideellen Interessen in den Bereichen medizinische Ausbildung, Weiterbildung und Fortbildung. Mit der Gründung der AOUC hat die lang entwickelte Idee als gemeinsames Referat der DGOU und des BVOU eine Form angenommen, die dem immer stärkeren $\mathrm{Zu}$ sammenwachsen der Fächer Orthopädie und Unfallchirurgie folgt. Das Zusammenrücken der Fachgesellschaften und des Berufsverbands zu einer gemeinsamen Akademie folgt dem gemeinsamen Ziel aller Beteiligten, $\mathrm{O}$ und $\mathrm{U}$ als ein starkes Fachgebiet zu repräsentieren.

Quelle

Akademie Orthopädie Unfallchirurgie, www.aouc.de 